\title{
Desenvolvimento de um Aplicativo Móvel para Fiscalização do Uso dos Recursos Hídricos (SIGFIS)
}

\author{
Lourivaldo J. F. C. Vasconcelos ${ }^{1}$, Hélvio A. L. Ferreira ${ }^{2}$, Jeísa P. O. Domingues ${ }^{3}$, \\ Marco A. O. Domingues ${ }^{1}$, Hernande P. Silva ${ }^{1}$ \\ ${ }^{1}$ Instituto Federal de Ciência e Tecnologia de Pernambuco - IFPE \\ ${ }^{2}$ Agência Pernambucana de Águas e Clima - APAC. \\ ${ }^{3}$ Departamento de Computação. Universidade Federal Rural de Pernambuco (UFRPE)

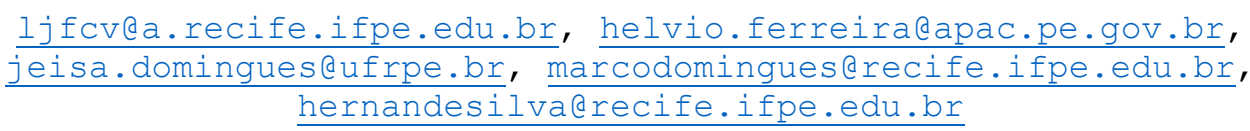

\begin{abstract}
The management of water resources has been undergoing constant transformations, making it increasingly necessary to develop tools to support management and inspection, with an educational and regulatory character. Based on this, this paper aims to present the development of an application that assists in the activity of monitoring the use of water resources with the use of geographic information systems. Entitled as SIGFIS, using an Android operating system with Kotlin programming language, with data in georeferenced formats and defined information plans, the application aims to assist tax agents in their field visits, in order to provide accuracy in assessments and agility in services, since it gathers relevant information for their work.
\end{abstract}

Resumo. A gestão dos recursos hídricos vem passando por transformações tornando urgente o desenvolvimento de ferramentas de apoio à gestão e fiscalização, com caráter educativo e regulador. Este artigo tem como objetivo apresentar o desenvolvimento de um aplicativo que auxilie na atividade de fiscalização dos recursos hídricos utilizando sistemas de informações geográficas. Intitulado SIGFIS, utilizando o sistema operacional Android com linguagem de programação Kotlin, com dados em formatos georreferenciados e planos de informações, o aplicativo visa auxiliar os agentes fiscais em suas visitas a campo, acurácia nas autuações e agilidade nos serviços, visto que reúne informações relevantes e imprescindíveis ao seu trabalho.

\section{Introdução}

Atualmente a temática ambiental vem sendo palco para diversos debates mundiais. A redução dos recursos naturais é um tema de preocupação global devido, principalmente, aos impactos causados pela industrialização e crescimento populacional. Neste sentido, a água é o bem essencial para a vida e para o processo de desenvolvimento das nações. Portanto, emerge a necessidade de promover um desenvolvimento sustentável aliado a uma gestão com ferramentas capazes de contribuir com processos de fiscalização, controle e uso consciente. 
Ao longo do tempo a gestão dos recursos hídricos vem passando por constantes transformações que vão desde disputas em relação ao uso até modelos de administração da infraestrutura hídrica. Conforme afirma Santos (2013, p. 12), "é necessário fazer uma gestão mais intensa dos recursos hídricos a fim de garantir a disponibilidade de água em seus diferentes tipos de uso".

Uma das ferramentas de gestão promovidas pelas políticas de recursos hídricos é a fiscalização do uso, com caráter educativo e regulador. Entretanto tais instrumentos necessitam de aperfeiçoamento, Miranda (2021). Um dos fortes aliados da gestão pública neste segmento é o sistema de informações geográficas.

Desta forma, este artigo tem como objetivo apresentar o desenvolvimento de um aplicativo voltado para um sistema de informações geográficas que auxilie na atividade de fiscalização do uso dos recursos hídricos, intitulado como SIGFIS.

Com esta intenção, será adotada uma metodologia com etapas voltadas para a coleta e criação de um banco de dados, seguidos por uma padronização e compatibilização destes dados. Aliado a isto, a revisão da legislação auxilia na geração de mapas temáticos com programa de geoprocessamento.

Como resultado espera-se que o SIGFIS possa contribuir com a obtenção de dados com maior precisão cartográfica, com melhorias nos procedimentos de fiscalização, facilitando os trabalhos de campo dos agentes afim de contribuir de forma efetiva com as tomadas de decisão, promovendo maior segurança jurídica aos processos de qualquer agência ou órgãos reguladores das águas.

\section{Gestão e políticas dos recursos hídricos}

Segundo dados da ONU, a escassez de água atinge milhões de pessoas mundialmente. Além da quantidade, a qualidade da água também é um fato preocupante, tanto que o número de pessoas que não tem acesso à água salubre passa de bilhões (UNESCO, 2009).

No Brasil, a importância com os recursos hídricos ainda é falha, mas está ganhando força. Como destaca Freitas (2008, p. 30), "o Brasil, nos últimos anos, vem tomando consciência do problema. Afinal, um povo que possui os maiores rios do mundo tem dificuldade em imaginar que pode ficar sem água."

Com a Constituição Federal de 1988, extinguiu-se o domínio privado e foi estabelecido que todos os corpos de água, a partir de outubro de 1988, passariam a ser de domínio público. Sendo assim, os recursos hídricos passaram a ser reconhecidos dentro da estrutura global do meio ambiente. Em janeiro de 1997, foi criada a Lei ${ }^{\circ}$ 9.433, também chamada como lei das águas, que estabelece a Política Nacional de Recursos Hídricos (PNRH) e prevê instrumentos de controle e gestão das águas (BRASIL, 1997).

Os fundamentos da Política Nacional dos Recursos Hídricos estão no artigo $1^{\circ}$ da Lei n. 9.433/97:

\footnotetext{
I - reconhecer a água como bem econômico e dar ao usuário uma indicação de seu real valor;

II - incentivar a racionalização do uso da água;

III - obter recursos financeiros para o financiamento dos programas e intervenções previstos nos planos de recursos hídricos. (BRASIL 1997, art. 1º)
}

Com a intenção de desenvolver mecanismos capazes de oferecer suporte administrativo e jurídico à gestão dos recursos, a Lei Federal no 9.433/97 instituiu o Sistema Nacional de Gerenciamento de Recursos Hídricos (SINGREH), que tem como objetivo coordenar o gerenciamento e implantação da PNRH visando preservar e 
recuperar os recursos hídricos. O sistema é composto por órgãos reguladores: o Conselho Nacional de Recursos Hídricos (CNRH); os Conselhos de Recursos Hídricos dos Estados e do Distrito Federal, os órgãos dos poderes públicos federal, estaduais, do Distrito Federal e municipais cujas competências se relacionem com a gestão de recursos hídricos; os Comitês de Bacias Hidrográficas (CBHs) (BRASIL, 1997).

A Política Nacional de Recursos Hídricos estabelece também instrumentos institucionais como os Planos de Recursos Hídricos, desenvolvidos para bacias hidrográficas, que prevê direitos e deveres no uso da água com ligação com o Sistema de Informação de Recursos Hídricos interligando estados e municípios com as ações e políticas públicas referentes à água (SIRVINSKAS, 2015).

Como afirma Souza Júnior (2004, p. 152), o uso de um modelo democrático de "gestão de recursos hídricos, no aspecto restrito à participação social, representa um avanço, conquanto outros países desenvolvidos possuem estruturas bastante centralizadas de gestão".

\section{Sistema de Informações Geográficas}

Ao longo dos anos o interesse pela informação geográfica tem sido uma constante. Segundo Cavalcante (2015), esse interesse é justificado pelo fato de ampliar e dar suporte a áreas tão distintas como as geociências, economia e gestão, sociologia e saúde, engenharias, planejamento e monitoramento espacial, entre outras.

Utilizada nas diversas áreas citadas, seu emprego permite ao usuário a correlação de variáveis distintas proporcionando análise, simulação e diversos cenários. Para tal, foram criadas ferramentas específicas de geoprocessamento chamadas de Sistema de Informações Geográficas (SIG), conhecido também por sua sigla em inglês GIS Geographic Information System, dotado de funcionalidades como: cartografia digital, GPS, sensoriamento remoto, aerofotogrametria, processamento digital de imagens, entre outros.

De acordo com Câmara \& Medeiros (1998, p. 48) as características dos dados geográficos permitem sua utilização como ferramentas para produção de mapas, suporte para análise espacial de fenômenos, ou como um banco de dados geográficos, com funções de armazenamento e recuperação de informação espacial. Nesse contexto, e levando em consideração a dimensão física e a localização espacial, o SIG é capaz de estabelecer relações topológicas existentes para armazenar e visualizar a topologia de um mapa, considerando vizinhança, através da relação de um sistema de coordenadas e seus atributos, proximidade e pertinência entre objetos geográficos.

\section{Dispositivos Móveis}

De acordo com a definição da UNESCO (2014), "Os dispositivos móveis incluem qualquer tecnologia portátil e conectada, como telefones celulares básicos, leitores eletrônicos, smartphones e tablets, além de tecnologias incorporadas como leitores de smartcard". Os principais dispositivos móveis são smartphones, tablets e notebooks. E se tornaram capazes de combinar funções de voz, texto, internet, aplicativos, pesquisa, redes sociais e "serviços pervasivos baseados em localização", segundo Dery, Kolb e Macornick (2014, p. 559). 


\subsection{Aplicativos para dispositivos móveis}

O rápido aumento no número de smartphones com recursos multimídias cada vez mais avançados, possibilitou aos usuários a utilização destes nas mais diversas tarefas. Grande parte das funcionalidades presentes nos aparelhos são possíveis graças aos aplicativos, chamados também de apps, softwares voltados especificamente para dispositivos móveis.

No mercado, há diversas plataformas para aparelhos celulares, como: Android (Google), IOS (Apple Inc), Windows Phone (Microsoft), etc. E para realização do desenvolvimento dos aplicativos, existem três abordagens distintas: desenvolvimento de aplicativos nativos, na qual o app é desenvolvido em plataforma específica e programado com linguagem nativa desse sistema; Progressive Web App (PWA) cuja implementação utiliza linguagens de programação para web. Tanto a interface como algumas funcionalidades se assemelham a uma aplicação móvel, podendo ser acessado por um navegador; e desenvolvimento de aplicativos híbridos, na qual os artefatos são desenvolvidos com linguagens e ferramentas da WEB e posteriormente convertido para o formato nativo. Essa característica permite que o mesmo código seja portado para diversos sistemas operacionais. Dessa maneira é possível obter mais produtividade e a curva de aprendizado é considerada menor em relação ao desenvolvimento nativo. A decisão sobre qual tipo de desenvolvimento utilizar dependerá dos recursos que se pretende utilizar.

O aplicativo que será apresentado como resultado deste trabalho foi desenvolvido no formato nativo, pois fornece melhor acesso aos recursos de hardware, melhor desempenho e integração facilitada com a ferramenta de visualização de mapas utilizada, além de existir possibilidade de utilizar o aparelho sem conexão à internet.

\subsection{Kotlin}

Kotlin é uma linguagem de programação orientada a objeto de código aberto e gratuito, desenvolvido pela empresa JetBrains, a mesma que criou a plataforma Android Studio. A Kotlin veio com objetivo de facilitar a programação em linguagem Java com a redução de codificação.

"O Kotlin é uma linguagem de programação moderna e estaticamente tipada usada por mais de $60 \%$ dos desenvolvedores Android profissionais. Seu uso ajuda a estimular a produtividade, a satisfação do desenvolvedor e a segurança do código." (Developers Android, 2021)

Devido às vantagens citadas anteriormente, o aplicativo resultado deste trabalho foi desenvolvido de forma nativa utilizando a linguagem Kotlin e o sistema operacional Android. Outros pontos que favoreceram a escolha da linguagem Kotlin foi a existência de exemplos no site oficial do Android e na documentação da ferramenta de exibição de mapas e conversão de código JAVA para Kotlin feita de forma automática pela IDE Android Studio.

\section{Android}

Uma das plataformas mais utilizadas em dispositivos móveis, o Android possui uma arquitetura flexível e de código aberto (open source). Funciona como uma pilha de softwares baseada no sistema Linux em um modelo de camadas: Kernel Linux, Camada de abstração de hardware, Android Runtime, Bibliotecas, estrutura da Java API e aplicativos do sistema. 
Dentre muitas as características que o Android possui, NICOLAI (2015) ressalta que "[...] o Android possui nativamente um framework de aplicações, uma máquina virtual Dalvik (Java) otimizada para dispositivos móveis, um navegador web baseado na engine de código aberto Webkit, suporte a arquivos de mídia de áudio e vídeo e um ambiente de desenvolvimento rico em ferramentas". Em relação aos aplicativos disponíveis para Android, Afanaci Junior (2012) afirma que o processo para se cadastrar é simples "Basta ter um cadastro com perfil de desenvolvedor, concordar com os termos de uso estipulados e pagar uma taxa de registro". Com o cadastro efetivado, o desenvolvedor terá acesso a envio de aplicações, configurações e monitoração dos dados relacionados aos aplicativos enviados.

\section{Etapas Adotadas}

Para o desenvolvimento do SIGFIS, foram adotadas as etapas descritas a seguir:

a) Inicialmente foi realizada uma revisão bibliográfica e documental a respeito da evolução da gestão dos recursos hídricos e políticas adotadas em âmbito nacional.

b) Para resultar em um aplicativo capaz de atender as expectativas propostas, foram coletados dados e informações através de reuniões realizadas com o departamento de informática da agência estadual de águas em conjunto com fiscais responsáveis pela gestão de recursos hídricos na região da pesquisa.

c) Com a coleta dos dados de arquivos em formatos georreferenciados, foi decidido que o aplicativo armazenaria em seu banco informações espaciais com formatos compatíveis com aplicações de geoprocessamento. Para o aplicativo do SIGFIS foi utilizado o programa de geoprocessamento de software livre QGIS (2020). Com o intuito de padronizar os dados, foi feita uma revisão cartográfica e optouse por trabalhar com o sistema de coordenadas de projeção geográfica (datum SIRGAS2000). Para esta etapa, os dados georreferenciados obtidos junto à equipe de fiscalização, possuíam diversas extensões, sendo todos convertidos para o ".shp" (referente a shapefile), por meio do QGIS.

d) Para a montagem dos planos de informações, que conceitualmente reúnem informações que se referem aos aspectos de uma região (GEODEN, 2021), foi realizado um estudo através de pesquisas bibliográficas e com as reuniões mencionadas anteriormente. Como consequência, foram definidas diretrizes de pesquisa, uma com base de dados cadastrais e temáticos: (municípios, bacias hidrográficas e hidrografia de rios e reservatórios); e outra com dados territoriais legalmente relevantes (processos de fiscalização, unidades de conservação, área de proteção dos mananciais, zoneamento de aquíferos, hidrogeologia, municípios em estiagem e águas de domínio da União).

\subsection{Modelagem do banco de dados}

O ponto inicial na modelagem do aplicativo trata da importação dos arquivos com os planos de informações para um banco de dados que possa ser reconhecido pelo aplicativo SIGFIS. Para tal, foi usado o banco de dados PostgreSQL com a extensão espacial PostGIS, e QGIS para processar os arquivos com informação geográfica .shp e realizar a conversão e importação no PostgreSQL.

Em seguida foi construído um sistema com a responsabilidade de realizar consulta nesse banco de dados e transformar o resultado para o formato "GeoJSON". Baseado em Json, este formato aberto permite representar informações geográficas através de formas 
como: pontos, linhas e polígonos com coordenadas geográficas, juntamente com seus atributos não-espaciais (EMTU). Assim, foi possível visualizar no SIGFIS todos os planos de informações elaborados e realizar atualizações a cada novo processo de fiscalização criado.

\subsubsection{Objetivos do aplicativo móvel SIGFIS}

Além de proporcionar apoio à gestão e fiscalização dos recursos hídricos por meio de um SIG, o aplicativo SIGFIS tem como meta auxiliar os agentes fiscais em suas visitas a campo, no sentido de proporcionar economia e agilidade nos serviços, visto que reúne informações relevantes e imprescindíveis ao seu trabalho. Sendo assim, os requisitos propostos para o aplicativo adotam os seguintes pontos:

a) Leitura do sensor GPS com o reconhecimento em tempo real do posicionamento do aparelho móvel;

b) Leitura dos planos de informações definidos no SIGFIS considerando o posicionamento do aparelho móvel;

c) Bufferização de áreas previstas para fiscalização em campo como forma de melhorar rendimento e economia de espaço de armazenamento do aparelho móvel;

d) Cadastro de usos de recursos hídricos identificados em campo como forma de registro inicial e auxiliar aos relatórios de fiscalização;

e) Alerta aos agentes fiscais no caso de fiscalização realizada em posicionamento inserido em algum território legalmente relevante.

\subsubsection{Tecnologias utilizadas}

A partir da definição dos objetivos a serem alcançados pelo aplicativo foram adotados recursos tecnológicos capazes de cumprir todas as etapas de criação e desenvolvimento, conforme segue:

a) Diagramação dos Recursos;

b) Servidor WEB;

c) Sistema operacional e Linguagem de Programação;

d) Interface de Programação de Aplicações (API) de Integração;

e) Sistema de Gerenciador de Banco de Dados;

f) API de Geolocalização.

Os usos das tecnologias citadas acima serão expostos no resultado obtido com o desenvolvimento do aplicativo.

\section{Implementação}

Com as bases de dados e os planos de informações definidos, traçadas a modelagem e as tecnologias utilizadas, tem-se como resultado a arquitetura do aplicativo e suas camadas.

Conforme a Figura 1 apresenta, o banco de dados utilizado foi o PostgreSQL com a API de geolocalização MapBox. Vale ressaltar que o PostgreSQL foi o repositório escolhido por ser um banco de dados relacional, ser utilizado por órgãos de fiscalização e poder ser aplicado facilmente em soluções geográficas. 


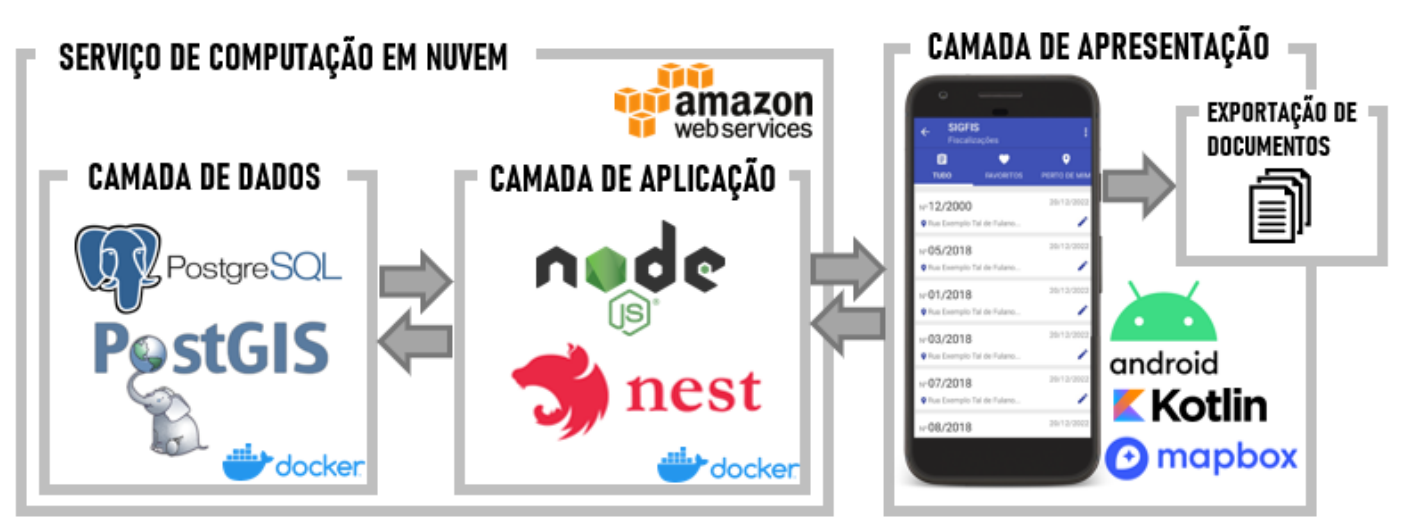

Figura 1. Arquitetura de desenvolvimento do aplicativo móvel SIGFIS

A arquitetura do aplicativo é dividida em três camadas:

a) Camada de Apresentação: responsável por interagir diretamente com o fiscal. Foi utilizado o sistema operacional Android e a linguagem de programação de código fonte aberto Kotlin, escolhida por se tratar de uma linguagem concisa e segura estaticamente tipada. A API de geolocalização utilizada foi o Mapbox que permite a interação de mapas quando utilizados por Android SDK em aplicativos.

b) Camada de Aplicação: responsável por intermediar a comunicação entre a camada de apresentação e de dados. Foi utilizado um servidor em nuvem na Amazon Web Services (AWS) em conjunto com a plataforma Docker e uma API na linguagem de programação NodeJS como a interface de comunicação. A opção pelo servidor e a plataforma proporcionam ao SIGFIS agilidade e rapidez nas aplicações e mobilidade quanto a ajustes necessários. Na API NodeJS foi configurada a segurança empregando o protocolo HTTPS, utilizada a instância com Ubuntu Server 18.04 LTS, que fornece 1GB de Memória de processamento, 8GB de Memória de armazenamento e $1 \mathrm{CPU}$, recursos suficientes para o protótipo do aplicativo.

c) Camada de Dados: responsável por armazenar os planos de informação e os dados das fiscalizações, através do servidor AWS. Foi adotado para o SIGFIS o sistema gerenciador de banco de dados relacional PostgreSQL versão 11.4-R1, optado por fornecer $1 \mathrm{~GB}$ de Memória de processamento, 20 GB de Memória de armazenamento e $1 \mathrm{CPU}$, recursos suficientes para o protótipo do aplicativo.

\subsection{Implementação do protótipo - SIGFIS}

Ao entrar no aplicativo, após a tela de carregamento pode-se observar, conforme Figura 2 , as telas de agendamento, listagem e busca de processos de fiscalização, e filtragem de fiscalizações na área atual do fiscal.

Na Figura 3, observa-se os planos de informações/mapas, informações de data de atualização do mapa, status de atualização, com opções de habilitar/desabilitar exibição.

É possível carregar o mapa de satélite com os planos de informações de hidrografia, municípios e processos de fiscalização, além de mapa de ruas. 


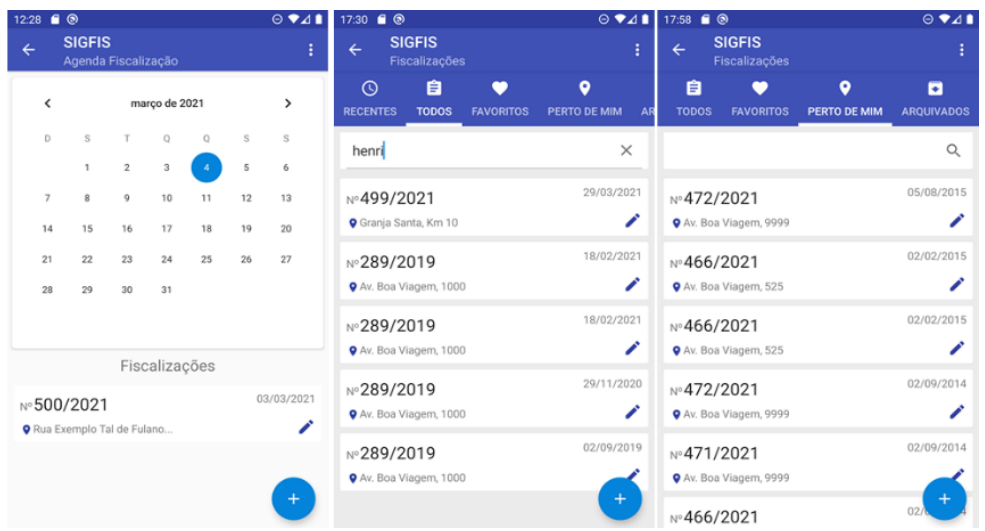

Figura 2. Fiscalizações e Agendamento

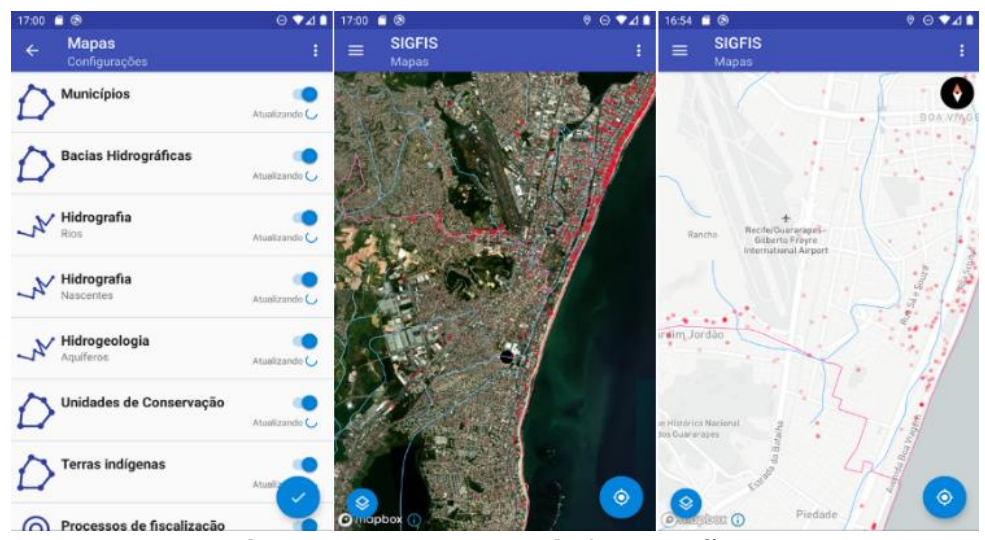

Figura 3. Planos de informações

Na Figura 4, visualiza-se a tela de registro de nova vistoria, com atalho para visualização de mapas do item da página anterior. Além das opções de gerar e exportar relatório, captura de fotos e áudios, cadastro de informações do usuário da água e cadastro de irregularidades observadas durante vistoria.

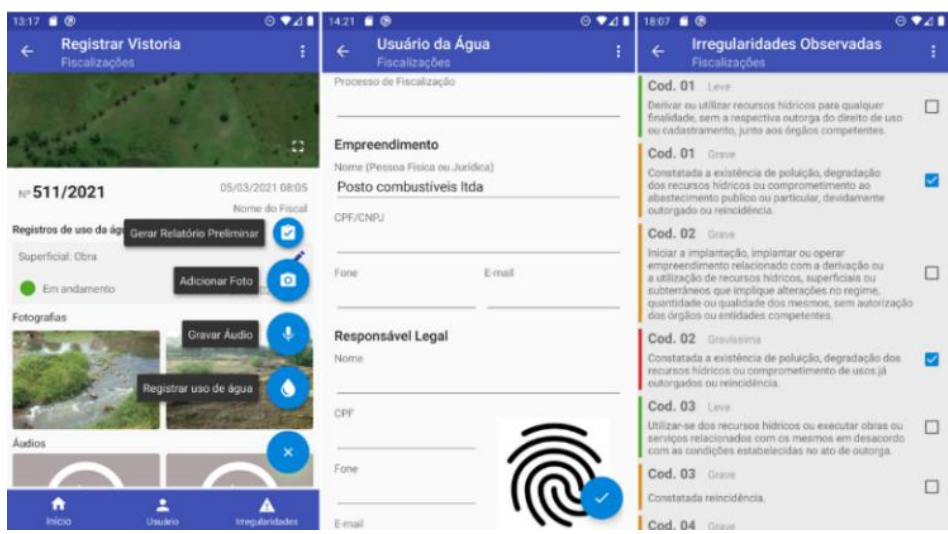

Figura 4. Registro de usuário e infrações

Na Figura 5, é possível visualizar a tela para cadastro de registro de uso de água, onde são selecionados a data e ponto no mapa. Nos formulários seguintes seleciona-se o tipo e subtipo de uso, que irá definir os próximos formulários a serem apresentados. No exemplo em questão, informa-se a situação e tipo de obra e dimensões, além das opções de busca por processos anteriores de fiscalização, com preenchimento dos dados do processo, outorga, em casos de processos existentes durante a busca. 


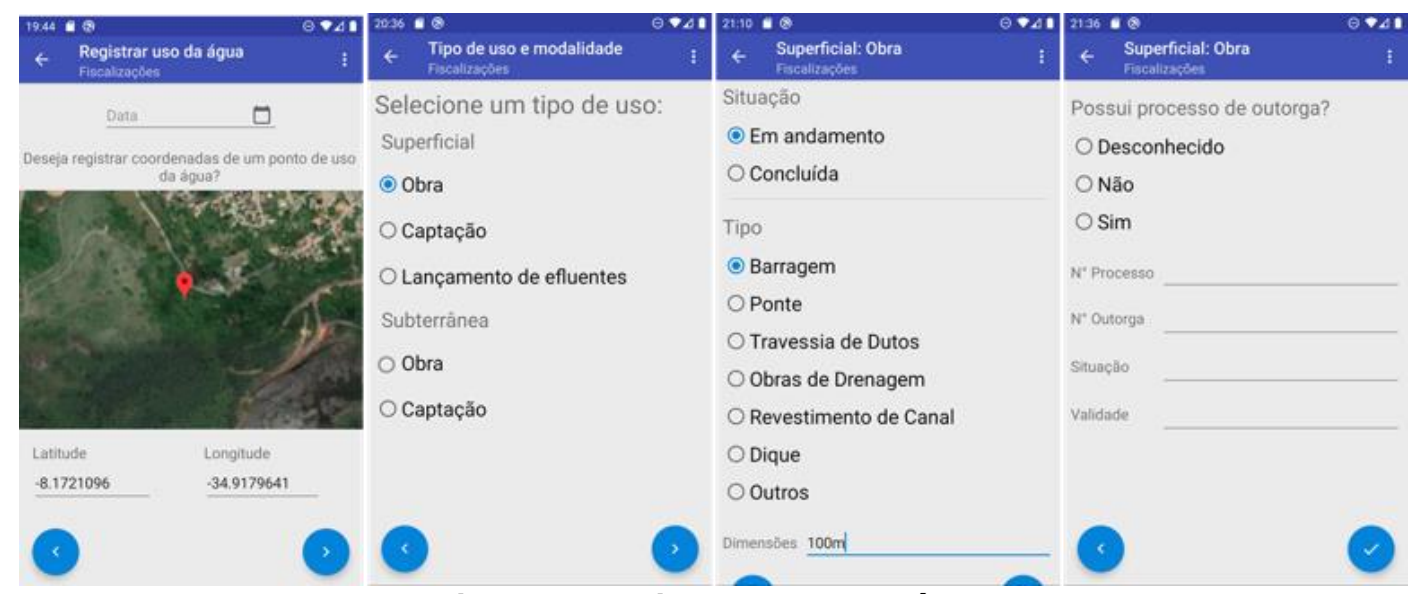

Figura 5. Registro de uso de água

\section{Considerações}

Observando os resultados obtidos por meio das pesquisas, análises e reuniões com potenciais usuários da solução, considera-se que os objetivos do desenvolvimento do SIGFIS foram alcançados. Durante simulações de uso o aplicativo proporcionou apoio à fiscalização dos recursos hídricos com agilidade e rapidez nos processos dos agentes fiscalizadores, diminuindo inclusive o retorno de visitas a campo, fato atrelado à possibilidade de validar informações geográficas durante a vistoria.

Como projeto futuro em relação ao uso do aplicativo pelos órgãos reguladores, o SIGFIS estará aberto a desenvolver aplicações em relação a impressões de documentos nos locais de vistoria com uso de impressoras portáteis, trazendo ainda mais agilidade aos fiscais.

Por fim a fiscalização dos recursos hídricos em conjunto com ferramentas de apoio fortalece a Política Nacional de Recursos Hídricos e contribui de forma efetiva com as tomadas de decisões.

\section{Referências}

Afanaci Junior, Marcelo. (2012) “Agreguei: sistema agregador de ofertas para plataforma Android.” 71 f. Trabalho de Conclusão de Curso (Especialização) - Universidade Tecnológica Federal do Paraná, Curitiba.

Brasil. (1997) “Lei n. 9.433, de 8 de janeiro de 1997.” http://www.camara.leg.br/legin/ fed/lei/1997/lei-9433-8-janeiro-1997-374778-norma-pl.html. Acesso em: 12 fev. 2021.

Brasil. (1998) "Constituição da República Federativa do Brasil, de 5 de outubro de 1988". https://www.planalto.gov.br/ccivil03/constituicao/constituicao.htm. Acesso em: 11 fev. 2021.

Câmara, Gilberto; Medeiros, José S. Princípios básicos em Geoprocessamento. In: ASSAD, Eduardo D.; SANO, Edson E. (1998) "Sistemas de Informações Geográficas. Aplicações na agricultura.” 2. ed. Brasília: Embrapa.

Cavalcante, Rodrigo. (2015) “Introdução ao SIG" Pró-Reitoria de Planejamento e Desenvolvimento. UFMG. https://www.ufmg.br/proplan/wp-content/uploads/Apostil 
a-de-Introdu\%C3\%A7\%C3\%A3o-ao-SIG-Proplan-2015.pdf. Acesso em: 02 abr. 2021.

Dery, Kristine; Kolb, Darl; Macornick, Judith. (2014) "Trabalhando com fluxo conectivo: como o uso de smartphones está evoluindo na prática”. European Journal of Information Systems.

Developers. (2021) "Abordagem Kotlin do Android." https://developer.android.com/ kotlin/first?hl=pt. Acesso em: 30 mar. 2021.

Developers. (2021) "Pilha de Software do Android." https://developer.android.com/gui de/platform? hl=pt-br. Acesso em: 28 mar. 2021.

EMTU. (2019) “Relatório Mensal Páginas Dados Abertos” https://www.emtu.sp.gov.br/ EMTU/pdf/RelatorioDadosAbertosEMTU_janeiro2019.pdf. Acesso em: 02 abr. 2021.

Freitas, Vladimir Passos de. (2008) "Águas - considerações gerais. Águas: aspectos jurídicos e ambientais". $7^{\circ}$ ed. Curitiba: Juruá.

Geoden (2021) "Sistema de Informação Geográfica - SIG” http://geoden.uff.br/ geoprocessamento/. Acesso em: 04 abr. 2021.

Miranda, Graziele Muniz. (2021). Motivações e desafios para a implementação da gestão integrada de recursos hídricos em federações. Revista De Gestão De Água Da América Latina, 17(2020), Revista de Gestão de Água da América Latina, 01 February 2021, Vol.17(2020).

Nicolai, Bruno Bernardeli et al. "Google android-a plataforma, seus componentes e suas versões." http://www.williamluis.com.br/wp-content/uploads/2013/10/TCC-GoogleAndroid-Final.pdf. Acesso em: 02 abr. 2021.

QGIS Development Team (2020) "QGIS Geographic Information System. Versão 3.16.5: Hannover" Open Source Geospatial Foundation Project, 2020. http://qgis.osgeo.org. Acesso em: 05 abr. 2021.

Sirvinskas, Luís Paulo. (2015) "Manual de direito ambiental.” 13. ed. Saraiva, São Paulo.

Santos, Rafael Rocha Rodrigues dos. (2013) "Sistema de Fiscalização da Agência Nacional de Águas" UnB. https://bdm.unb.br/bitstream/10483/6705/1/2013Rafael RochaRodriguesDosSantos.pdf. Acesso em: 02 abr. 2021.

Souza Junior, Wilson Cabral de. (2004) "Gestão das Águas no Brasil: reflexões, diagnósticos e desafios.” São Paulo: Petrópolis.

Unesco. (2014) "O Futuro da Aprendizagem Móvel: Implicações para planejadores e gestores de políticas.” https://unesdoc.unesco.org/ark:/48223/ pf0000228074. Acesso em: 05 mar. 2021.

Unesco. (2009) "Programa Mundial de Avaliação da Água das Nações Unidas: Gestão Integrada de Recursos Hídricos em ação Paris." https://unesdoc.unesco.org/ark:/ 48223/pf0000181891. Acesso em: 18 fev. 2021. 